\title{
Effects of Dry Needling on Connectivity of Corticospinal Tract, Spasticity, and Function of Upper Extremity in People with Stroke: Study Protocol for a Randomized Controlled Trial
}

\author{
Masoome Ebrahimzadeh ${ }^{1}$, Noureddin Nakhostin Ansari ${ }^{2,3}$, Iraj Abdollahi ${ }^{1, *}$, Behnam Akhbari ${ }^{1}$, \\ Saeideh Monjezi ${ }^{4}$ \\ ${ }^{1}$ Department of Physiotherapy, University of Social Welfare and Rehabilitation Sciences, Tehran, Iran \\ ${ }^{2}$ Department of Physiotherapy, School of Rehabilitation, Tehran University of Medical Sciences, Tehran, Iran \\ ${ }^{3}$ Research Center for War-affected People, Tehran University of Medical Sciences, Tehran, Iran \\ 4Musculoskeletal Rehabilitation Research Center, Ahvaz Jundishapur University of Medical Sciences, Ahvaz, Iran
}

Received January 20, 2021

Revised August 16, 2021

Accepted September 5, 2021

Correspondence to

Iraj Abdollahi

Department of Physiotherapy,

University of Social Welfare and

Rehabilitation Sciences, Tehran, Iran

E-mail irajabdollahi@hotmail.com
Background: Spasticity is a common motor disorder in adult stroke patients. Injury to the corticospinal tract (CST) is associated with spasticity. Dry needling (DN) has positive impacts on spasticity reduction and improvement in the range of motion (ROM) in stroke patients. This study aims to investigate the effectiveness of DN on the connectivity of the CST and the level of spasticity in adult stroke patients.

Methods: This double-blind randomized controlled trial will enroll and randomly assign stroke patients to either the experimental group, for receiving three sessions of DN for the spastic wrist flexors, or the control group, for sham needling. The primary outcome measures will be diffusion tensor imaging and the Modified Modified Ashworth Scale score to assess CST connectivity and wrist flexor spasticity, respectively. The Box and Block Test and standard goniometry are the secondary outcome measures to assess hand dexterity and active and passive wrist ROM, respectively. Measurements will be taken both before and after the intervention.

Discussion: The results of this study will provide important evidence of the effects of DN on CST connectivity, spasticity, and arm function in adult stroke patients.

Trial registration: This trial is registered in the Iranian Registry of Clinical Trials (IRCT) (https://www.irct.ir; IRCT20191208045649N1).

Keywords: Stroke, Dry needling, Muscle spasticity, Corticospinal tract, Diffusion tensor imaging

\section{INTRODUCTION}

Stroke is a major neurological disease with a high prevalence and, every year, more than 13.7 million individuals suffer an incident stroke [1]. Stroke is a leading cause of disability in adults worldwide [2], and patients suffer poststroke neurological deficits such as hemiparesis, spasticity, and ataxia. Hemiparesis significantly affects the ability of stroke patients to perform voluntary movements and undertake activities of daily living.

The corticospinal tract (CST) is a major pathway for skilled voluntary movements. Post-stroke CST damage leads to ataxia and loss of fine motor control of the more affected upper extremity [3]. Following a stroke, the neural drive to the muscles is impaired, and CST connectivity is reduced [4]. Thus, post-stroke CST damage leads to both weakness and muscle spasticity, both of which contribute to the development of post-stroke movement disorders.

Spasticity is a common post-stroke motor disorder. Lance defined spasticity as a motor disorder characterized by a velocity-dependent increase in muscle tone due to hyperexcitability of the stretch reflex [5]. The prevalence of post-stroke spasticity is $30 \%$ to $80 \%$ [6]. Although spasticity is a common symptom in stroke patients, the mechanisms 
underlying this disorder are poorly elucidated. Both neural and mechanical mechanisms have been suggested as sources of muscle spasticity in stroke [6,7]. Spasticity impedes the initiation of active movement due to a hyperactive stretch reflex [8]. Therefore, it follows that spasticity should be controlled and managed to decrease complications and to improve voluntary activities in stroke patients.

Dry needling (DN) has been recently used to control poststroke spasticity. A systematic review revealed that DN has positive effects on spasticity, pain, and range of motion (ROM) [9]. A systematic review and meta-analysis of seven studies found moderate evidence of the positive effects of DN on spasticity [10].

A prospective case report on the effects of $\mathrm{DN}$ on spastic muscles, including flexor carpi radialis (FCR), flexor carpi ulnaris (FCU), and pronator teres, of a stroke patient confirmed that both spasticity and alpha motor neuron excitability improved, and that the patient could voluntarily extend the more affected wrist and fingers after DN [11]. A single group clinical trial with 29 stroke patients found that DN improved spasticity, alpha motor neuron excitability, active wrist extension, and hand dexterity [12]. Another study reported significant improvements in post-stroke spasticity as well as active abduction and external rotation of the shoulder [13]. Previous studies have suggested that central effects of DN play a role in the improvements observed in the spasticity of stroke patients $[11,12]$. Improvements in spasticity and active voluntary movements after DN of spastic muscles as well as post-DN increases in brain activity [14] led us to hypothesize that these improvements in stroke patients are associated with an improvement in CST connectivity. To test this hypothesis, we aim to investigate whether DN of spastic wrist flexors of stroke patients will improve CST connectivity, visualized using diffusion tensor imaging (DTI), and active voluntary wrist extension on the more affected side.

\section{MATERIALS AND METHODS}

\section{Study design}

This study protocol is a double-blind, randomized controlled trial (RCT) based on the Standard Protocol Items: Recommendations for Interventional Trials (SPIRIT) guideline $[15,16]$. The trial is registered at the Iranian Registry of Clinical Trials (IRCT; https://www.irct.ir: IRCT20191208 045649N1). This study protocol has been approved by the Ethics Committee of the University of Social Welfare and Rehabilitation Sciences (USWRS; approval ID: IR.USWR. REC.1398.149, on November 25, 2019). This RCT will follow the Consolidated Standards of Reporting Trials (CONSORT) for reporting the study's results [17].

\section{Informed consent}

Eligible patients will be verbally informed about the purpose and procedures of the study by the examining physiotherapist, and written informed consent will be obtained from the participants prior to their enrolment in the study. Patients will be allowed to withdraw from the study at any time during the study.

\section{Study population}

Stroke patients referred to the physiotherapy clinic and fulfilling the predefined eligibility criteria will be enrolled in this trial from November 2021 to December 2022.

\section{Inclusion and exclusion criteria}

Inclusion criteria: 1) age 18-65 years; 2) incident stroke with hemiplegia; 3) post-stroke duration of at least 6 months; 4) wrist flexor Modified Modified Ashworth Scale (MMAS) spasticity score $\geq 1 ; 5$ ) not receiving antispastic drug $24 \mathrm{~h}$ before study enrolment; and 6) ability to understand and follow instructions.

Exclusion criteria: 1) presence of other neurological diseases; 2) fixed muscle contracture at the wrist joint; and 3) concurrent participation in other studies.

\section{Study procedures}

This study will be conducted at the National Brain Mapping Laboratory, Tehran, Iran. Patients will be referred from the Physiotherapy Clinic of the USWRS. Eligible patients willing to participate in the study will be randomly assigned to either the experimental group or the control group after recording their demographics and baseline assessments. The outcome measures will be randomly applied by an experienced physiotherapist blinded to the group allocation. A flowchart of the study's timeline is shown in Fig. 1.

The participants' files will be preserved in a locked cabinet with a restricted access. All data will be cleared, coded, and electronically entered into a password-protected computer laptop for analysis. Double-checked data entry will be conducted to ensure data accuracy and integrity.

\section{Randomization, allocation, and blinding}

Participants will be assigned to the groups according to the stratified permuted block randomization method. The Random Allocation software (1.1 version, Isfahan University of Medical Sciences, Isfahan Province, Iran) will be used in blocks with random sizes of four and six patients. The blocks will be concealed by sealed opaque envelopes. The randomization process will be managed by an independent statistician who is not involved in the study in order to minimize selection bias and ensure allocation concealment $[18,19]$. The envelopes will only be opened after informed 


\begin{tabular}{|c|c|c|c|c|}
\hline & \multicolumn{4}{|c|}{ Study period } \\
\hline & \multirow{2}{*}{$\begin{array}{c}\text { Enrollment } \\
-t_{1}\end{array}$} & \multirow{2}{*}{$\frac{\text { Allocation }}{t_{0}}$} & \multicolumn{2}{|c|}{ Post-allocation } \\
\hline Timepoint** & & & $t_{1}$ & $t_{2}$ \\
\hline \multicolumn{5}{|l|}{ Enrollment: } \\
\hline Eligibility screen & $\mathrm{x}$ & & & \\
\hline \multirow{2}{*}{$\begin{array}{l}\text { Informed consent } \\
\text { Demographics } \\
\text { questionnaire }\end{array}$} & & $\mathrm{x}$ & & \\
\hline & & $\mathrm{x}$ & & \\
\hline Allocation & & $\mathrm{x}$ & & \\
\hline \multicolumn{5}{|l|}{ Interventions: } \\
\hline Dry needling & & & $x$ & \\
\hline Sham needling & & & $x$ & \\
\hline \multicolumn{5}{|l|}{ Assessments: } \\
\hline FA & & $x$ & & $x$ \\
\hline \multirow{3}{*}{$\begin{array}{c}\text { MMAS } \\
\text { Wrist active range } \\
\text { of extension } \\
\text { Wrist passive range } \\
\text { of extension }\end{array}$} & & $x$ & & $x$ \\
\hline & & $\mathrm{X}$ & & $x$ \\
\hline & & $\mathrm{x}$ & & $\mathrm{X}$ \\
\hline BBT & & $x$ & & $x$ \\
\hline
\end{tabular}

Fig. 1. The SPIRIT Schematic diagram of study schedule. Standard Protocol Items: Recommendation For Interventional Trial; $F A=$ fractional anisotropy; $M M A S=$ modified modified ashworth scale; BBT $=$ Box and Block Test; $-t_{1}=$ Pre-Study, Screening/Consent; $t_{0}=$ Pre-study Baseline/Randomization; $t_{1}$ $=$ Study, Intervention; $\mathrm{t}_{2}=$ After Intervention.

consent and baseline measurements have been obtained.

The intervention will be delivered by an experienced and trained physiotherapist and would then be assessed by another physiotherapist who will be blinded to the group allocation. Participants will be blinded to the group allocation.

\section{Sample size}

We computed the sample size based on previous findings of the effects of DN in stroke patients [12]. Considering the non-normal distribution of data, a total sample size of 106 (n $=53$ in each group) was calculated for the primary outcome of fractional anisotropy (FA) measure of DTI using the Wilcoxon-Mann-Whitney test, medium effect size of $0.5, \alpha$ level of 0.05 , and power of 0.8 .

\section{Intervention}

Interventions will be carried out at the Physiotherapy Clinic of the USWRS (Tehran, Iran). All procedures will be performed between 9:00 and 12:00 AM. Participants in the experimental group will receive three sessions of $\mathrm{DN}$ for the more affected upper limb, every other day. In this study, the wrist flexors, FCU and FCR, will be needled in accordance with the Standards for Reporting Interventions in Clinical Trials of Acupuncture (STRICTA) guideline [20]. DN will be performed by a trained physiotherapist with more than 10 years' experience in using $\mathrm{DN}$.

\section{1) Dry needling}

DN will be performed using a sterile, stainless, and disposable needle (size $0.25 \times 30 \mathrm{~mm}$; DongBangAcuPrime Ltd., Korea) in accordance with the STRICTA 2010 checklist. Participants will lie in a supine position, with the treatment arm at a $45^{\circ}$ abduction at the shoulder, and with the elbow in extension and supination. For each muscle, one needle will be deeply $(20-25 \mathrm{~mm})$ inserted using the cone-shaped, fastin and fast-out technique at approximately $1 \mathrm{~Hz}$ frequency for 1 minute. The acupoints for DN of FCR and FCU are 1 $\mathrm{cm}$ medial to the midpoint and $4 \mathrm{~cm}$ below the elbow crease and the midpoint of the proximal third of a line connecting the medial epicondyle to the styloid process of the ulna, respectively $[11,12]$. The points needled are approximate motor points of muscles. No other treatment will be administered.

\section{2) Sham needle}

Patients allocated to the control group will receive the same protocol provided to the experimental group except that a sham needle with a thick point will be applied to the same muscles to stimulate the skin without piercing it [21].

\section{Outcome measures}

The primary outcomes are the connectivity of CST and the level of spasticity. The secondary outcomes are active and passive range of motion (ROM) of wrist extension and hand dexterity, which will be assessed both before and after the intervention.

\section{1) Connectivity of CST}

Changes in the connectivity of the CST will be determined by measuring the FA. In the white matter, water diffusion occurs at maximum speed along the nerve fibers; therefore, its diffusion is anisotropic. Degrees of anisotropic diffusion of the water molecule vary between 0 (completely isotropic) and 1 (completely anisotropic). A larger value indicates greater connectivity of the CST $[22,23]$. DTI is a new magnetic resonance imaging (MRI) method that is used for mapping 
the process of diffusion of water molecules in biological tissues [24]. Patients will lie in a supine and relaxed position in a 3/0-T MRI system (Siemens Prisma, Germany, Erlangen) equipped with 6 channel head coils [25]. The DTI will be performed by an expert technician, and the data will be analyzed by a brain imaging specialist.

\section{2) Level of spasticity}

The spasticity level of the wrist flexors will be determined by the MMAS, which is a reliable and valid tool for assessing post-stroke spasticity and grades spasticity from "0" (no increase in muscle tone) to "4" (affected part rigid in flexion or extension) points [26-28]. The Persian version of the MMAS will be used to grade the severity of wrist flexor spasticity [29]. Assessment will be performed with the patient is in a supine and relaxed position with their arms placed alongside the body, head in midline position, and the affected forearm in mid-position. The wrist will be moved passively from the maximum flexion to the maximum extension counting 1001 [27].

\section{3) Hand dexterity}

Hand dexterity will be assessed by a reliable and valid Box and Block Test (BBT) [30,31]. The BBT includes a box, which is divided into two equal parts by a partition, and contains 150 pieces. The box will be placed in front of the participants and they will be instructed to pick up the pieces one by one using the affected hand and to put them into the other part of the box as quickly as possible. The number of pieces transferred from one part to the other within $60 \mathrm{~s}$ will be recorded as the BBT score.

\section{4) Active and passive wrist extension}

A standard goniometer will be used for measuring the active and passive ROM of the wrist extension. Participants will sit on a comfortable chair and the elbow will be positioned on the table in 90-degree flexion without any rotation. The goniometer axis will be located on the snuff box, the stationary arm will be located along the outer border of the radius, and the movable arm will be located in alignment with the $2^{\text {nd }}$ metacarpal bone. For assessing the passive ROM, the assessor will passively extend the wrist and record the end position of wrist extension. The same procedure will be followed to assess the active ROM of wrist extension by asking the patient to perform a voluntary extension of the wrist [12].

\section{Data monitoring}

An independent committee will supervise the study procedure to ensure the clarity and accuracy of data.

\section{Statistical analysis}

SPSS version 22.0 will be used for data analysis. Descriptive statistics will be calculated for all the demographics and clinical measures. An analysis of covariance (ANCOVA) will be used to compare the intergroup post-intervention scores for continuous variables. Baseline scores, the strokeonset duration, and age will be considered as covariates for reducing the effects of confounding variables. The KruskalWallis test will be used to determine intergroup differences on the ordinal dependent variable of the MMAS. Effect size will be calculated using Cohen's d coefficient. A $p$-value lower than 0.05 will be considered statistically significant.

\section{DISCUSSION}

The results of this RCT will provide important evidence of the effects of DN in revealing changes in the CST in stroke patients. Although various descending motor tracts possibly contribute to voluntary movements, the CST is a major descending motor pathway that contributes to the control of voluntary movements. To our knowledge, this study, for the first time, will measure the connectivity of the CST after DN using DTI to detect improvements. DTI is an imaging method that can reveal the level of recovery of neural pathways, such as CST. In this study, the FA measure of DTI, which has high sensitivity to small structural changes in neural pathways, will be measured [32].

Neuromodulation strategies to reduce muscle spasticity, such as DN, are hypothesized to enhance the engagement of the descending motor CST in stroke patients. The recovery of active movements after DN of post-stroke spastic muscles $[6,8]$ may be indicative of CST connectivity. Therefore, it follows that potential improvements in CTS connectivity after DN will explain the positive outcomes on the level of spasticity as well as active movements and function of the more affected hand [31].

In post-stroke hemiplegia with spasticity, the balance between the facilitatory influence of medial reticulospinal tract (RST) and the inhibitory influence of the dorsal RST is lost [33]. In stroke patients, severe hemiparesis and impairments in motor function are associated with enhanced reticulospinal activity [34-36]. Therefore, it may be postulated that, concomitant with improvements in CST connectivity, the contribution of facilitatory reticulospinal inputs to spastic muscles might be modulated after $\mathrm{DN}$ and could result in a reduction of spasticity. This hypothesis needs to be further tested in future investigations.

The strength of the study is that a double-blind RCT with a sham control design will be conducted. The random allocation of participants will help to minimize potential confounder variables and the risk of bias in the interpretation 
of the results. Nonetheless, we anticipate difficulties in participant recruitment and retention in this study due to the prevailing COVID-19 pandemic crisis in Iran. Therefore, only some stroke patients may be interested to participate and engage in this study. In order to improve the recruitment of subjects to achieve the target sample size, we will develop a list of stroke patients from various neurorehabilitation clinics to telephonically contact them about their possible participation in the study. As an incentive, we will offer financial payment for the cost of taxi service [37]. However, we anticipate that participant accrual in this RCT will be slower than planned. A potential limitation of the study should be noted. This study will evaluate the short-term effects of DN. Long-term follow-up, unfortunately, is unfeasible in the present study because of the COVID-19 pandemic crisis, limited time to conduct the study, and financial restrictions.

\section{ACKNOWLEDGEMENTS}

This study protocol is submitted in partial fulfillment of the requirements for a $\mathrm{PhD}$ degree in Physiotherapy for Masoome Ebrahimzadeh from the University of Social Welfare and Rehabilitation Sciences, Tehran, Iran.

\section{AUTHORS' CONTRIBUTIONS}

NNA developed the study concept and hypothesis. NNA, IA, ME, BA, and SM designed the study protocol. ME drafted the first version of this manuscript and NNA reviewed and revised the manuscript critically for intellectual content. All authors have read, commented, and approved the final manuscript for submission.

\section{CONFLICT OF INTEREST}

The authors declare no conflict of interest.

\section{ORCID}

Masoome Ebrahimzadeh, https://orcid.org/0000-0003-2462-2309

Noureddin Nakhostin Ansari, https://orcid.org/0000-0003-2742-2273

Iraj Abdollahi, https://orcid.org/0000-0002-4256-8513 Behnam Akhbari, https://orcid.org/0000-0003-0586-2534 Saeideh Monjezi, https://orcid.org/0000-0001-9963-8108

\section{REFERENCES}

1. Lindsay MP, Norrving B, Sacco RL, Brainin M, Hacke W, Martins S, et al. World Stroke Organization (WSO): global stroke fact sheet 2019. Int J Stroke 2019;14:806-17.

2. Katan M, Luft A. Global burden of stroke. Semin Neurol 2018; 38:208-11.

3. Jaspers E, Byblow WD, Feys H, Wenderoth N. The corticospinal tract: a biomarker to categorize upper limb functional potential in unilateral cerebral palsy. Front Pediatr 2016;3:112.

4. Bowden JL, Taylor JL, McNulty PA. Voluntary activation is reduced in both the more- and less-affected upper limbs after unilateral stroke. Front Neurol 2014;5:239.

5. Lance JW. What is spasticity? Lancet 1990;335:606.

6. Kuo CL, Hu GC. Post-stroke spasticity: a review of epidemiology, pathophysiology, and treatments. Int J Gerontol 2018;12: 280-4.

7. Lieber RL, Steinman S, Barash IA, Chambers H. Structural and functional changes in spastic skeletal muscle. Muscle Nerve 2004;29:615-27.

8. Nielsen JB, Crone C, Hultborn H. The spinal pathophysiology of spasticity--from a basic science point of view. Acta Physiol (Oxf) 2007;189:171-80.

9. Valencia-Chulián R, Heredia-Rizo AM, Moral-Munoz JA, Lucena-Anton D, Luque-Moreno C. Dry needling for the management of spasticity, pain, and range of movement in adults after stroke: a systematic review. Complement Ther Med 2020;52:102515.

10. Fernández-de-Las-Peñas C, Pérez-Bellmunt A, LlurdaAlmuzara L, Plaza-Manzano G, De-la-Llave-Rincón AI, Navarro-Santana MJ. Is dry needling effective for the management of spasticity, pain, and motor function in post-stroke patients? A systematic review and meta-analysis. Pain Med 2021;22:131-41.

11. Ansari NN, Naghdi S, Fakhari Z, Radinmehr H, Hasson S. Dry needling for the treatment of poststroke muscle spasticity: a prospective case report. NeuroRehabilitation 2015;36:61-5.

12. Fakhari Z, Ansari NN, Naghdi S, Mansouri K, Radinmehr H. A single group, pretest-posttest clinical trial for the effects of dry needling on wrist flexors spasticity after stroke. NeuroRehabilitation 2017;40:325-36.

13. Mendigutia-Gómez A, Martín-Hernández C, Salom-Moreno J, Fernández-de-Las-Peñas C. Effect of dry needling on spasticity, shoulder range of motion, and pressure pain sensitivity in patients with stroke: a crossover study. J Manipulative Physiol Ther 2016;39:348-58.

14. Mohammadpour F, Ali Oghabian M, Nakhostin Ansari N, Naghdi S, Dommerholt J. Effects of dry needling on post-stroke brain activity and muscle spasticity of the upper limb: a case report. Acupunct Med 2021;39:69-71.

15. Chan AW, Tetzlaff JM, Altman DG, Laupacis A, Gøtzsche PC, Krleža-Jerić K, et al. SPIRIT 2013 statement: defining standard protocol items for clinical trials. Ann Intern Med 2013;158:200-7.

16. Chan AW, Tetzlaff JM, Gøtzsche PC, Altman DG, Mann H, Berlin JA, et al. SPIRIT 2013 explanation and elaboration: 
guidance for protocols of clinical trials. BMJ 2013;346:e7586.

17. Moher D, Hopewell S, Schulz KF, Montori V, Gøtzsche PC, Devereaux PJ, et al. [CONSORT 2010 Explanation and Elaboration: updated guidelines for reporting parallel group randomised trials (Chinese version)]. Zhong Xi Yi Jie He Xue Bao 2010;8:701-41. Chinese.

18. Doig GS, Simpson F. Randomization and allocation concealment: a practical guide for researchers. J Crit Care 2005;20:18791; discussion 191-3.

19. Matts JP, Lachin JM. Properties of permuted-block randomization in clinical trials. Control Clin Trials 1988;9:327-44.

20. MacPherson H, Altman DG, Hammerschlag R, Youping L, Taixiang W, White A, et al. Revised STandards for Reporting Interventions in Clinical Trials of Acupuncture (STRICTA): extending the CONSORT statement. PLoS Med 2010;7:e1000261.

21. Tekin L, Akarsu S, Durmuş O, Cakar E, Dinçer U, Kıralp MZ. The effect of dry needling in the treatment of myofascial pain syndrome: a randomized double-blinded placebo-controlled trial. Clin Rheumatol 2013;32:309-15.

22. Chabert S, Scifo P. Diffusion signal in magnetic resonance imaging: origin and interpretation in neurosciences. Biol Res 2007;40:385-400

23. Puig Alcántara J. Diffusion tensor imaging in acute ischemic stroke: the role of anisotropy in determining the time of onset and predicting long-term motor outcome [dissertation]. Girona: Universitat de Girona; 2014. 112 p.

24. Welniarz Q, Dusart I, Roze E. The corticospinal tract: evolution, development, and human disorders. Dev Neurobiol 2017;77:81029.

25. Yoo YJ, Kim JW, Kim JS, Hong BY, Lee KB, Lim SH. Corticospinal tract integrity and long-term hand function prognosis in patients with stroke. Front Neurol 2019;10:374.

26. Naghdi S, Ansari NN, Mansouri K, Asgari A, Olyaei GR, Kazemnejad A. Neurophysiological examination of the Modified Modified Ashworth Scale (MMAS) in patients with wrist flexor spasticity after stroke. Electromyogr Clin Neurophysiol 2008;48:35-41.

27. Naghdi S, Ansari NN, Azarnia S, Kazemnejad A. Interrater reliability of the Modified Modified Ashworth Scale (MMAS) for patients with wrist flexor muscle spasticity. Physiother Theory Pract 2008;24:372-9.
28. Ansari NN, Naghdi S, Hasson S, Fakhari Z, Mashayekhi M, Herasi M. Assessing the reliability of the Modified Modified Ashworth Scale between two physiotherapists in adult patients with hemiplegia. NeuroRehabilitation 2009;25:235-40.

29. Nakhostin Ansari N, Naghdi S, Forogh B, Hasson S, Atashband M, Lashgari E. Development of the Persian version of the Modified Modified Ashworth Scale: translation, adaptation, and examination of interrater and intrarater reliability in patients with poststroke elbow flexor spasticity. Disabil Rehabil 2012;34:1843-7.

30. Desrosiers J, Bravo G, Hébert R, Dutil E, Mercier L. Validation of the Box and Block Test as a measure of dexterity of elderly people: reliability, validity, and norms studies. Arch Phys Med Rehabil 1994;75:751-5.

31. Platz T, Pinkowski C, van Wijck F, Kim IH, di Bella P, Johnson G. Reliability and validity of arm function assessment with standardized guidelines for the Fugl-Meyer Test, Action Research Arm Test and Box and Block Test: a multicentre study. Clin Rehabil 2005;19:404-11.

32. Kumar P, Kathuria P, Nair P, Prasad K. Prediction of upper limb motor recovery after subacute ischemic stroke using diffusion tensor imaging: a systematic review and meta-analysis. J Stroke 2016;18:50-9.

33. Ganguly J, Kulshreshtha D, Almotiri M, Jog M. Muscle tone physiology and abnormalities. Toxins (Basel) 2021;13:282.

34. Choudhury S, Shobhana A, Singh R, Sen D, Anand SS, Shubham $S$, et al. The relationship between enhanced reticulospinal outflow and upper limb function in chronic stroke patients. Neurorehabil Neural Repair 2019;33:375-83.

35. McPherson JG, Chen A, Ellis MD, Yao J, Heckman CJ, Dewald JPA. Progressive recruitment of contralesional cortico-reticulospinal pathways drives motor impairment post stroke. J Physiol 2018;596:1211-25.

36. Owen M, Ingo C, Dewald JPA. Upper extremity motor impairments and microstructural changes in bulbospinal pathways in chronic hemiparetic stroke. Front Neurol 2017;8:257.

37. Treweek S, Lockhart P, Pitkethly M, Cook JA, Kjeldstrøm M, Johansen M, et al. Methods to improve recruitment to randomised controlled trials: Cochrane systematic review and metaanalysis. BMJ Open 2013;3:e002360. 